\title{
TRUNCATION OF THE BINARY DISTRIBUTION FUNCTION IN GLOBULAR CLUSTER FORMATION
}

\author{
E.VESPERINI \\ Scuola Normale Superiore \\ Piazza dei Cavalieri 7, 56126 Pisa-Italy \\ AND \\ D.F. CHERNOFF \\ Department of Astronomy \\ Space Science Building, Cornell University 14853 Ithaca, (New \\ York) USA
}

\section{Introduction}

Recent observational searches suggest that the frequency of primordial binaries in globular clusters may reach $\sim 10 \%$ (see Hut et al. 1992 for a review). Several different treatments conclude that primordial binaries are effective in halting core collapse, supporting the core and driving the postcore collapse expansion phase (Goodman \& Hut 1989, McMillan et al. 1990, 1991, Gao et al. 1991, Heggie \& Aarseth 1992, McMillan \& Hut 1994). The abundance and binding energy distribution have a direct impact on observable characteristics of globular clusters such as the size of the core radius (Vesperini \& Chernoff 1994). In this analysis we have investigated how the initial binary distribution function may be altered in the formation of a cluster; the key question we have addressed is whether it is possible for binaries, assumed to be primordial, to survive the birth of the cluster. A detailed description of our results is in Vesperini \& Chernoff (1995).

\section{Results}

We focus on an assumed phase of violent relaxation immediately after a large, tidally truncated gas cloud has formed stars. If the initial ratio of kinetic to potential energy of the system is smaller than $1 / 2$ then the gross 
properties (radius, velocity dispersion) of the system undergo damped oscillations until a virialized state is reached. When binaries are present and suffer collisional interactions within the cluster, their internal binding energy is an additional sink or source for changes in stellar translational energy. For the available binding energy to play a dynamical role in the cluster evolution, binaries must interact collisionally. Collisional interactions occur most rapidly at the point of maximum contraction when the background stellar density and velocity dispersion are largest. As the velocity dispersion reaches very high values at the point of maximum contraction, even binaries hard in the virialized system are soft and can be disrupted more easily.

Using $N$-body simulations and analytic calculations we have examined the binary destruction process during violent relaxation. The main conclusions are:

1. There is no significant change in the gross cluster properties at the end of violent relaxation due to the interaction between internal (binary) degrees of freedom and translational degrees of freedom.

2. It is possible to identify a characteristic binding energy below which the initial distribution is truncated. This cut-off binding energy, $\epsilon_{c u t}$, scales with the number of particles in the system as $\epsilon_{\text {cut }} \sim N^{-1.19}$ and the hardness parameter for the cut-off energy as calculated in the virialized system falls in all cases in the soft regime $(x<1)$.

3. Analytical calculations are in good agreement with results from $N$ body simulations and show that ionization is the main destructive process.

4. Some hardening of binaries with $\epsilon>\epsilon_{c u t}$ is observed in the $N$-body simulations. This is not described by the thermally averaged rate coefficients. Cold initial conditions used for the simulations mean some binaries are bound to the closest single particle. An interaction between the single particle and the binary is responsible for at least part of the observed hardening.

\section{References}

Gao B., Goodman J., Cohn H., Murphy B. (1991) Ap.J. 370, 567

Goodman J., Hut P. (1989) Nature 339, 40

Heggie D.C., Aarseth S.J. (1992) MNRAS 257, 513

Hut P., McMillan S.L., Goodman J. et al. (1992) Publ. Astron. Soc. Pacific 104, 981

McMillan S., Hut P., Makino J. (1990) Ap.J. 362, 522

McMillan S., Hut P., Makino J. (1991) Ap.J. 372, 111

McMillan S., Hut P. (1994) Ap.J. 427, 793

Vesperini E., Chernoff D.F (1994) Ap.J. 431, 231

Vesperini E., Chernoff D.F. (1995) Ap.J. in press 